\title{
Mechanical and Optical Properties of Waterborne UV Curing Coating Modified by Silica
}

\author{
Xiao-Xing YAN ${ }^{1,2, a,{ }^{*},}$, Yun-Ting CAI ${ }^{1, b}$, Guo-Yue $X U^{2, c}$ and Wei $X U^{1, d}$ \\ ${ }^{1}$ College of Furniture and Industrial Design, Nanjing Forestry University, Nanjing 210037, PR China \\ ${ }^{2}$ College of Material Science \& Technology, Nanjing University of Aeronautics and Astronautics, \\ Nanjing 211100, PR China \\ a yanxiaoxing@nuaa.edu.cn, ${ }^{b} 616940816 @ q q . c o m,{ }^{c}$ xuguoy@nuaa.edu.cn, ${ }^{d}$ xuwei@njfu.edu.cn \\ ${ }^{*}$ Corresponding author Tel.: +86-25-85427528; Fax: +86-25-85427528. E-mail: \\ yanxiaoxing@nuaa.edu.cn
}

Keywords: Modification, Waterborne UV wood coating, Mechanical, Gloss

\begin{abstract}
Silica $\left(\mathrm{SiO}_{2}\right)$ was used to modify the waterborne ultraviolet (UV) curing wood coatings, and the waterborne UV curing wood coating was prepared by optimizing the process parameters. The mechanical properties of the waterborne UV curing wood coating (hardness, adhesion and impact strength) and gloss were tested. When the $\mathrm{SiO}_{2}$ content in the waterborne UV curing wood coating was $3 \%$, the waterborne UV curing wood coatings was drying at $40^{\circ} \mathrm{C}$ for $30 \mathrm{~min}$ in the oven firstly, then irradiated with UV lamp for 1 min, the obtained waterborne UV curing wood coating had good hardness, adhesion and impact strength. However, when $\mathrm{SiO}_{2}$ content of waterborne UV curing wood coating was more than $3 \%$, the mechanical properties decreased. The gloss test showed that the gloss of waterborne UV curing wood coating was elevated with the decreased content of $\mathrm{SiO}_{2}$. When the $\mathrm{SiO}_{2}$ content was higher than $3 \%$, the gloss of waterborne UV curing wood coating exhibited matt.
\end{abstract}

\section{Introduction}

Wood coating was decorated on the surface of the wood products, and protected the surface of wood products [1]. The traditional wood coatings contain the large amounts of volatile organic compound (such as toluene, xylene, etc.) which had serious impacts on human health and environment [2]. In recent years, the waterborne UV curing coatings have the property of environmentally friendly, high curing speed and saving energy [3]. Therefore, the waterborne UV curing coatings meet the environmental protection requirements and have a great potential for development [4]. The one of the important part of the coating is gloss [5]. The high gloss of coating would cause certain damage to eyesight, therefore, more and more people prefer to low gloss products, such as matt gloss. There are a lot of ways for waterborne UV curing coatings to obtain ideal gloss, such as adding the matting agent [6], changing the formula composition and curing conditions, etc [7]. At present, the application of waterborne UV curing coatings often gets serious limits such as poor mechanical properties and the disadvantage of high gloss [8]. Therefore, the modified waterborne UV curing wood coatings could improve the mechanical properties and get ideal matte gloss.

The character of silica $\left(\mathrm{SiO}_{2}\right)$ is high specific surface area, inorganic rigid, infrared reflection, etc [9]. The added $\mathrm{SiO}_{2}$ to the coating can improve the thermal aging ability of coating, the ultraviolet aging resistance, and scratch resistance [10]. In this paper, $\mathrm{SiO}_{2}$ was modified to waterborne UV curing wood coating, improved the mechanical properties and achieved matte gloss through orthogonal experiments to optimize the composition and curing conditions. 


\section{Experimental}

\section{Materials}

All reagents were the analysis pure in the present experiment, which were directly used without further treatment. Waterborne UV curing wood coating was provided by Guangdong Yihua Timber Industry Co., LTD. Modifier $\mathrm{SiO}_{2}$ was provided by Shanghai Chemical Reagent Co., LTD.

\section{Coating Preparation}

In the preliminary experiments, when the quality ratio of $\mathrm{SiO}_{2}$ to waterborne UV curing wood coating was $1-5 \%$, the drying time was $20-40$ min and the number of UV lamps was $1-3$, it was found that the curing time is very long when the waterborne UV curing wood coating was only cured with UV curing lamp. Therefore, waterborne UV curing wood coating was first put in the oven at different temperature, and then dried under UV light.

Firstly, a certain amount of waterborne UV curing wood coating was poured out to the disposable paper cup. Then the amount of pre-designed $\mathrm{SiO}_{2}$ and waterborne UV curing coatings (the quality of the $\mathrm{SiO}_{2}$ to the waterborne UV curing coatings was 1.0-5.0\%) were measured respectively, and mixed. Finally, waterborne UV curing wood coating was coated on the surface of flat wood substrate. The coated wood substrates were firstly put in the oven at $40-50^{\circ} \mathrm{C}$ for $30 \mathrm{~min}$, then transferred to the UV curing machine for radiation curing with medium pressure mercury UV lamp. The distance of the lamp to coated wood substrates was $30 \mathrm{~cm}$, the number of UV lamps was 2 and the UV light curing time was 1-3 min.

\section{Characterization of Coating}

The impact strength of waterborne UV curing coatings was measured with the hammer according to GB/T1732-93. The adhesion of waterborne UV curing coatings was measured using the method of cross lattice adhesion test according to GB 9286-1998. The hardness of waterborne UV curing coatings was measured using hardness pencil according to GB 6739-86. The gloss of waterborne UV curing coatings was measured using gloss meter for testing.

\section{Results and Discussion}

\section{Orthogonal Experimental Analysis}

In this experiment, the main factors influencing the modified waterborne UV curing wood coating are $\mathrm{SiO}_{2}$ content, oven temperature and the UV radiation time. When these three factors were optimized, through orthogonal test the mechanical properties of the modified coating would be best. The orthogonal experiment was used to optimize the three factors affecting the performance of modified waterborne UV curing wood coating. Based on statistics theory and the orthogonal theory, the orthogonal experiment is a kind of multi-factor experiment method, which spends less time to solve the complex problems with the low cost. In the orthogonal experiments, the scope of the amount of three components was: the content of $\mathrm{SiO}_{2}(\mathrm{~A}): 1.0-5.0 \%$, oven temperature (B): $40-50^{\circ} \mathrm{C}$, the UV radiation time (C): 1-3min. Two levels were chose in each factor. The first level of factor $\mathrm{A}$ is $\mathrm{SiO}_{2}$ content of $1.0 \%$, and the second level of factor $\mathrm{A}$ is $\mathrm{SiO}_{2}$ content of $5.0 \%$. The first level of factor $\mathrm{B}$ is oven temperature of $40^{\circ} \mathrm{C}$, and the second level of factor $\mathrm{B}$ is oven temperature of $50^{\circ} \mathrm{C}$. The first level of factor $\mathrm{C}$ was $\mathrm{UV}$ radiation time of $1 \mathrm{~min}$, and the second level of factor $\mathrm{C}$ is the UV radiation time of $3 \mathrm{~min}$.

The content of $\mathrm{SiO}_{2}$, oven temperature, and the UV radiation time had a certain degree of influence on the mechanical properties of waterborne UV curing wood coatings such as adhesion, impact strength and hardness. By comparison, the gloss effect is more apparent. Therefore, in the orthogonal experiments, we focused on the analysis of the effect of modification conditions on the glossiness, then on this basis the process parameters was further optimized and the effect of modification on the mechanical performance was studied. 
In the Table 1, the average value for the first level (I) and the second level (II) of the these factors was calculate. Table 1 shows the three factors affect waterborne UV curing wood coatings gloss. Were calculated in the same column, The range $(R)$ was calculated with the same factors corresponding to the maximum average gloss and minimum average glossiness, and the variance was also calculated listed in Table 1. In general, the impact is greater if $\mathrm{R}$ and variance are greater. By comparing the $\mathrm{R}$ value and variance, it can be found that the effect of content of $\mathrm{SiO}_{2}$ on gloss of modified waterborne UV curing wood coating is the most obvious. The effect of the UV radiation time and oven temperature on the coating gloss is small. For energy saving, oven temperature was fixed at $40^{\circ} \mathrm{C}$, and UV radiation time was fixed for $1 \mathrm{~min}$ in the following experiments.

Table $1 \mathrm{SiO}_{2}$ modified table of orthogonal experiment

\begin{tabular}{ccccc}
\hline Sample & $\begin{array}{c}\text { Content of } \mathrm{SiO}_{2} \\
(\%)\end{array}$ & $\begin{array}{c}\text { Oven temperature } \\
\left({ }^{\circ} \mathrm{C}\right)\end{array}$ & $\begin{array}{c}\text { UV radiation time } \\
(\text { min })\end{array}$ & $\begin{array}{c}\text { Gloss } \\
(\%)\end{array}$ \\
\hline $1 \#$ & 1.0 & 40 & 3 & 54 \\
$2 \#$ & 1.0 & 50 & 1 & 56 \\
$3 \#$ & 5.0 & 40 & 1 & 10 \\
$4 \#$ & 5.0 & 50 & 3 & \\
I & 55.0 & 32.0 & 31.5 & \\
II & 9.5 & 0.5 & 33.0 & \\
R & 45.5 & 0.25 & 1.5 & \\
Variance & 2070.25 & & 2.25 & \\
\hline
\end{tabular}

\section{Effect of SiO2 Content on the Mechanical Properties of Waterborne UV Curable Wood Coating}

In order to research the key role of $\mathrm{SiO}_{2}$, on the basis of orthogonal experiment, a series of independent experiment was conducted: the oven temperature was fixed at $40^{\circ} \mathrm{C}$, the $\mathrm{UV}$ radiation time was fixed for $1 \mathrm{~min}$, and the content of $\mathrm{SiO}_{2}$ was changed to 0, 0.5\%, 1.0\%, 2.0\%, 3.0\%, 4.0\% and $5.0 \%$, respectively. It was found that $\mathrm{SiO}_{2}$ content had a certain effect on the hardness of waterborne UV curing wood coating. Fig. 1 shows $\mathrm{SiO}_{2}$ content on the influence of hardness of waterborne UV curing wood coating.

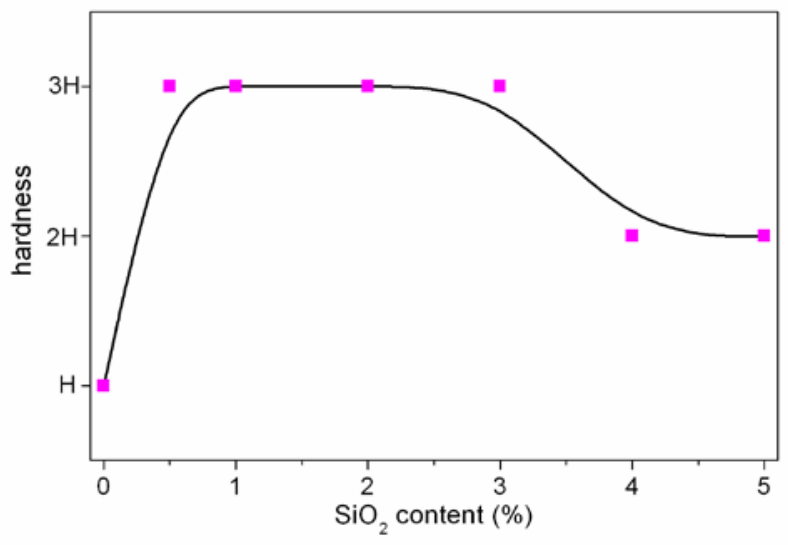

Fig. 1 Effect of $\mathrm{SiO}_{2}$ content on the hardness of waterborne UV curable wood coating

It can be seen from Fig. 1 that when the $\mathrm{SiO}_{2}$ content increased from 0 to $0.5 \%$, the hardness of waterborne UV curing wood coating increased from $\mathrm{H}$ to $3 \mathrm{H}$. Further increase of the $\mathrm{SiO}_{2}$ content to $3.0 \%$, the coating hardness is still maintaining high value of $3 \mathrm{H}$. This is because $\mathrm{SiO}_{2}$ particles have discrete distribution under the low content, and $\mathrm{SiO}_{2}$ particles can be spread evenly in waterborne UV curing wood coating, and $\mathrm{SiO}_{2}$ particles itself is rigid inorganic material. Therefore, the addition of $\mathrm{SiO}_{2}$ had greatly increased the hardness of waterborne UV curing wood coating. But when the $\mathrm{SiO}_{2}$ content was more than 3.0\%, the hardness of waterborne UV curing wood coating decreased. This is 
due to excessive $\mathrm{SiO}_{2}$ reunite between particles, and binding force of water-borne UV curing wood coating decreased, resulting in the decline of hardness [11].

Adhesion of coating is coating adhesion on the substrate under the specified load. Fig. 2 shows the relationship between the $\mathrm{SiO}_{2}$ content and adhesion. With the increase of $\mathrm{SiO}_{2}$ content adhesion presents "U" shaped curve. It can be seen from Fig. 2, with $\mathrm{SiO}_{2}$ content increased from 0 to $0.5 \%$, adhesion changed from grade 3 to grade 1 . When the added $\mathrm{SiO}_{2}$ content was increased to 3.0\%, coating has a better adhesion of grade 1. This is because the coating cohesion and mechanical interlocking increased with the increase of $\mathrm{SiO}_{2}$ content, leading to the increased adhesion. However, further increase of $\mathrm{SiO}_{2}$ content from 3.0\% to 5.0\%, adhesion has been down to level 2. $\mathrm{SiO}_{2} \mathrm{particles}$ will be gathered when the $\mathrm{SiO}_{2}$ content increased, and $\mathrm{SiO}_{2}$ particles cannot be evenly dispersed, which leads to the decreased adhesion [12]. In addition, the coating of elasticity will be reduced with the increase of $\mathrm{SiO}_{2}$ content, which reduces the consumption of energy [13]. Therefore, these leads to more easily broken coating, and the adhesion fell to level 2.

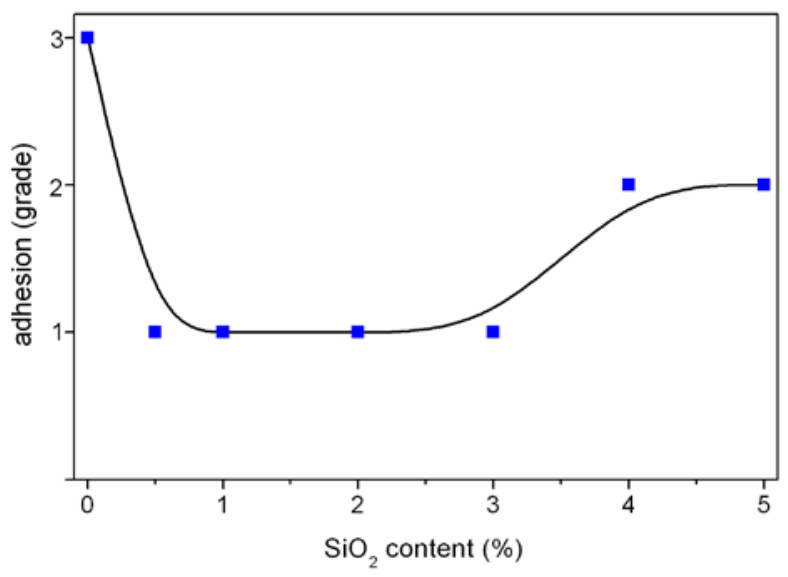

Fig. 2 Effect of SiO2 content on the adhesion of waterborne UV curable wood coating

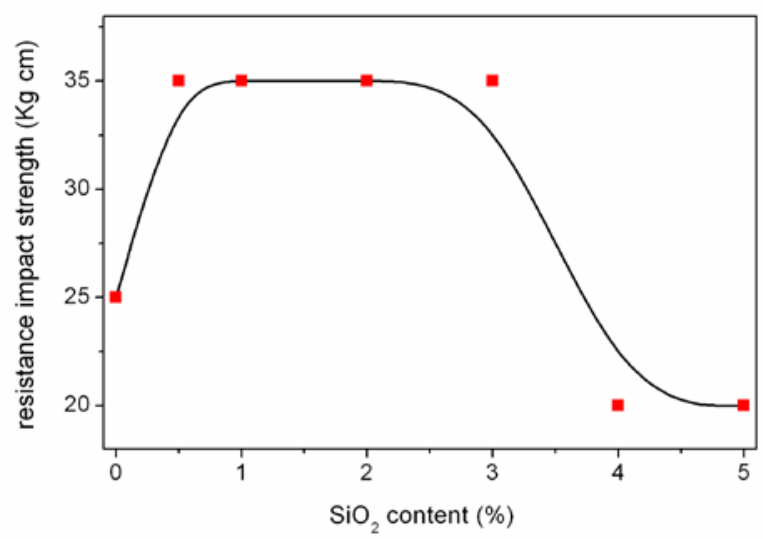

Fig. 3 Effect of SiO2 content on the impact strength of waterborne UV curable wood coating

In order to investigate the fatigue behavior of coating, after adhesion test, the impact resistance properties of the coating was measured, as shown in Fig. 3. Impact experiment investigated the dynamic load capacity of the coating. As seen from Fig. 3, when the $\mathrm{SiO}_{2}$ content increased from 0 to $0.5 \%$, the impact strength increased from $25 \mathrm{Kg} \cdot \mathrm{cm}$ to $35 \mathrm{Kg} \cdot \mathrm{cm}$. When the content of $\mathrm{SiO}_{2}$ further increased from $0.5 \%$ to $0.5 \%$, the impact strength of waterborne UV curing wood coating was 35 $\mathrm{Kg} \cdot \mathrm{cm}$. When the $\mathrm{SiO}_{2}$ content continued to increase, the impact resistance of waterborne UV curing wood coating was worse. The change trend is consistent with the change trend of adhesion, showed that the adhesion of waterborne UV curing wood coating is in direct proportion to its impact strength [14]. 


\section{Effect of SiO2 Content on the Gloss of Waterborne UV Curable Wood Coating}

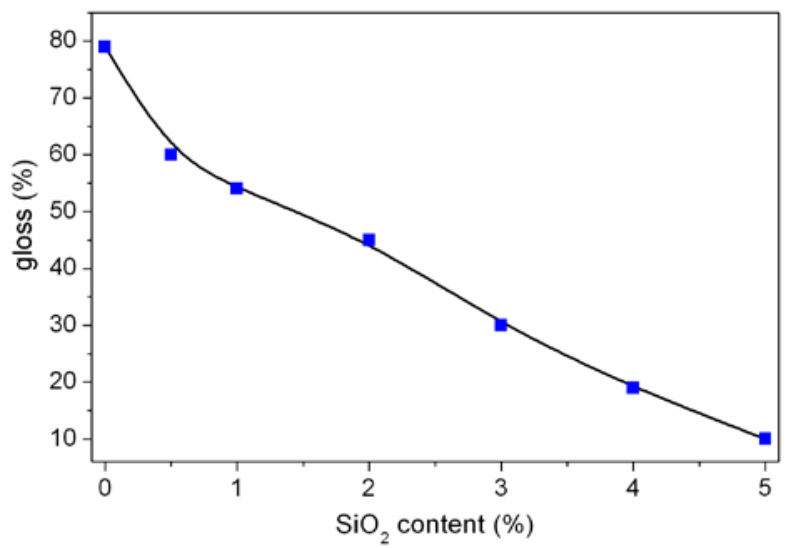

Fig. 4 Effect of SiO2 content on the gloss of waterborne UV curable wood coating

The impact of $\mathrm{SiO}_{2}$ content on gloss of waterborne UV curing wood coating was shown in Fig. 4 . As seen from Fig. 4, the gloss of waterborne UV curing wood coating was decreased with the increase of $\mathrm{SiO}_{2}$ content. When the $\mathrm{SiO}_{2}$ content increased from 0 to 3\%, the gloss of waterborne UV curing wood coating decreased significantly from $79.0 \%$ to $30.0 \%$. As $\mathrm{SiO}_{2}$ content continued to increase from $3.0 \%$ to $5.0 \%$, the gloss of waterborne UV curing wood coating continued to slowly decline from $30.0 \%$ to $10.0 \%$, and always kept the lower value. $\mathrm{SiO}_{2}$ particles do not dissolve in waterborne UV curing wood coating. With the increasing of $\mathrm{SiO}_{2}$ content, therefore, volume concentration of $\mathrm{SiO}_{2}$ particles on the coating surface increases and the coating surface has the fine roughness. When incident light reach the surface, scattering occurs and extinction, which reduces the gloss of waterborne UV curing wood coating. When the $\mathrm{SiO}_{2}$ content increased to a certain value, $\mathrm{SiO}_{2}$ particles gathered under high level, and the phenomenon of reunion could be seen on the coating surface, therefore, the gloss of waterborne UV curing wood coating decreased slowly. According to China wood furniture common technical standards GB/T 3324-3324, matte gloss value is below 35\%. As can be seen from the Fig. 4, when the $\mathrm{SiO}_{2}$ content was more than $3.0 \%$, waterborne UV curing wood coating showed matte gloss.

\section{Conclusion}

Orthogonal experiments showed that the mechanical properties of waterborne UV curing wood coating depended largely on the content of $\mathrm{SiO}_{2}$. The results of the influence of $\mathrm{SiO}_{2}$ on water-borne UV curing wood coatings mechanical properties showed that when the oven temperature was fixed at $40^{\circ} \mathrm{C}$, the UV radiation time fixed for $1 \mathrm{~min}$ and $\mathrm{SiO}_{2}$ content increased from 0 to $3.0 \%$, the hardness of waterborne UV curing wood coating increased from $\mathrm{H}$ to $3 \mathrm{H}$, adhesion increased from 3 level to 1 level, and the impact strength increased from $25 \mathrm{Kg} \cdot \mathrm{cm}$ and $35 \mathrm{Kg} \cdot \mathrm{cm}$, at this time the waterborne UV curing wood coating would have the best mechanical properties. But when the $\mathrm{SiO}_{2}$ content was more than 3.0\%, the hardness, adhesion and impact resistance of waterborne UV curing wood coating decreased. Gloss results showed that the gloss of waterborne UV curing wood coating decreased with the increase of $\mathrm{SiO}_{2}$ content. When the $\mathrm{SiO}_{2}$ content was greater than 3.0\%, waterborne UV curing wood coating showed matte gloss.

\section{Acknowledgement}

This research was financially supported by the Natural Science Foundation of Jiangsu Province (BK20150887), Specialized Research Fund for the Doctoral Program of Higher Education (20123204120019), University Natural Science Research Project of Jiangsu Province (14KJB220007), Jiangsu Planned Projects for Postdoctoral Research Funds (1402006A), China 
Postdoctoral Science Foundation funded project (2015M570444), Priority Academic Program Development of Jiangsu Higher Education Institutions (PAPD) and Jiangsu Overseas Research \& Training Program for University Prominent Young \& Middle-aged Teachers and Presidents.

\section{References}

[1] A. Kaboorania, N. Auclair, B. Riedl, et al, Physical and morphological properties of UV-cured cellulosenanocrystal (CNC) based nanocomposite coatings for wood furniture, Prog. Org. Coat. 93 (2016) 17-22.

[2] K.Q. Zhang, X.H. Li, Y.H. Zhao, et al, UV-curable POSS-fluorinated methacrylate diblock copolymers foricephobic coatings, Prog. Org. Coat. 93 (2016) 87-96.

[3] T. Zhang, W.J. Wu, X.J. Wang, Effect of average functionality on properties of UV-curable waterborne polyurethane-acrylate, Prog. Org. Coat. 68 (2010) 201-207.

[4] S.W. Zhang, A.X. Yu, X.Q. Song, et al, Synthesis and characterization of waterborne UV-curable polyurethane nanocomposites based on the macromonomer surface modification of colloidal silica, Prog. Org. Coat. 76 (2013) 1032-1039.

[5] V. Jancovicova, M. Mikula, B. Havlinova, et al, Influence of UV-curing conditions on polymerization kinetics and gloss of urethane acrylate coatings, Prog. Org. Coat. 76 (2013) 432-438.

[6] S. Rossi, M. Fedel, F. Deflorian, et al, Influence of different colour pigments on the properties of powder deposited organic coatings, Mater. Design 50 (2013) 332-341.

[7] G. Pia, E.C. Corcione, R. Striani, et al, Thermal conductivity of porous stones treated with UV light-cured hybrid organic-inorganic methacrylic-based coating. Experimental and fractal modeling procedure, Prog. Org. Coat. 94 (2016) 105-115.

[8] S.M. Mirabedini, M. Mohseni, S. PazokiFard, et al, Effect of $\mathrm{TiO}_{2}$ on the mechanical and adhesion properties of RTV silicone elastomer coatings, Colloids Surf. A 317 (2008) 80-86.

[9] L. Chen, R.G. Song, X.W. Li, et al, The improvement of corrosion resistance of fluoropolymer coatings by $\mathrm{SiO}_{2}$ /poly(styrene-co-butyl acrylate) nanocomposite particles, Appl. Surf. Sci. 353 (2015) 254-262.

[10] S.W. Zhang, Z.D. Chen, M. Guo, et al, Synthesis and characterization of waterborne UV-curablepolyurethane modified with side-chain triethoxysilaneand colloidal silica, Colloids Surf. A: Physicochem. Eng. Asp. 468 (2015) 1-9.

[11] B. Fiorentino, R. Fulchiron, V. Bounor-Legare, et al, Chemical modification routes of synthetic talc: Influence on its nucleating power and on its dispersion state, Appl. Surf. Sci., 109-110 (2015) 107-118.

[12] F. Mirshahi, S. Bastani, M.G. Sari, Studying the effect of hyperbranched polymer modification in the kinetics of curing reactions and physical/mechanical properties of UV-curable coatings, Prog. Org. Coat. 90 (2016) 187-199.

[13] J.Y. Dai, S.Q. Ma, Y.G. Wu, et al, High bio-based content waterborne UV-curable coatings with excellent adhesion and flexibility, Prog. Org. Coat. 87 (2015) 197-203.

[14] X.X. Yan, G.Y. Xu, Corrosion and mechanical properties of epoxy-polyurethane/bronze composite coatings with low infrared emissivity, Surf. Coat. Technol. 205 (2010) 2307-2312. 